Review Article

\title{
Current Status and Underlying Problems of Eradication of Poliomyelitis in the Remaining Endemic Countries
}

\author{
Anthony Chibuogwu Ike ${ }^{1,2, ~ *, ~ E l e a z a r ~ E m m a n u e l ~ R e w a r d ~}{ }^{1}$, Emmanuel Msugh Mbaawuaga ${ }^{2}$, \\ Obianuju Obiajulu Nnabuife ${ }^{1}$, Ibuchukwu Nkeonyenasoya Alexandra Orabueze ${ }^{1,3}$ \\ ${ }^{1}$ Department of Microbiology, University of Nigeria, Nsukka, Nigeria \\ ${ }^{2}$ Department of Biological Sciences, Benue State University, Makurdi, Nigeria \\ ${ }^{3}$ Department of Medical Microbiology, College of Medicine, University of Nigeria Enugu Campus, Enugu, Nigeria
}

Email address:

anthonyc.ike@unn.edu.ng (A. C. Ike)

${ }^{*}$ Corresponding author

\section{To cite this article:}

Anthony Chibuogwu Ike, Eleazar Emmanuel Reward, Emmanuel Msugh Mbaawuaga, Obianuju Obiajulu Nnabuife, Ibuchukwu Nkeonyenasoya Alexandra Orabueze. Current Status and Underlying Problems of Eradication of Poliomyelitis in the Remaining Endemic Countries. European Journal of Preventive Medicine. Vol. 6, No. 1, 2018, pp. 23-28. doi: 10.11648/j.ejpm.20180601.15

Received: February 6, 2018; Accepted: February 28, 2018; Published: March 15, 2018

\begin{abstract}
In 1988, the World Health Assembly of the World Health Organization (WHO) launched the Global Polio Eradication Initiative (GPEI), with the resolve to interrupt transmission of wild poliovirus (WPV) worldwide by the year 2000. Eighteen years after the intended year of eradication, that target is yet to be achieved. To investigate the reasons behind this, a check of recent publications on poliomyelitis and polio virus was carried out. The databases and websites of institutions and organizations involved in the polio eradication programme were also checked. The findings showed that although the goal of interrupting polio transmission has not been meet, a lot of progress has been made. By 2006, transmission of WPV had been interrupted in all but four countries: Afghanistan, India, Nigeria and Pakistan. In February 2012, India was delisted from the list of polio-endemic countries leaving the remaining 3 countries as the only endemic countries in the world. Wild type 2 and 3 viruses have both been eliminated from circulations. The number of polio cases in the remaining endemic countries has reduced drastically. Nigeria is close to the target, but is yet to be certified polio-free, having experienced a setback in 2016. However, as long as transmission of WPV continues in these countries, the world cannot be free from the disease, as these countries serve as reservoirs of infection and sources of export of the virus to other countries. To be able to achieve the target of interrupting the transmission of WPV in the remaining endemic countries, the identified problems, particularly conflict in the countries concerned, will have to be factored into the programmes geared toward eradication of the disease.
\end{abstract}

Keywords: Poliomyelitis, Poliovirus, Eradication, Wild Type, Endemic, Afghanistan, Pakistan, Nigeria

\section{Introduction}

Despite persistent global actions, poliomyelitis is yet to be eradicated. Giant strides have been taken in the quest to make the world polio-free. Currently, there are only two countries of the world in which polio remains endemic: Pakistan and Afghanistan [1]. There was detection of polio in Nigeria in 2016 after the last detected case in $2014[2,3]$. These countries serve as exporters of the virus to other parts of the world where polio has been eradicated thus necessitating the need for polio to be stamped out in these countries before there can be certification of a polio-free world. Through the Global Polio Eradication Initiative (GPEI) of the World Health Organization (WHO), there has been a significant drop of $99 \%$ in the number of detected polio cases from 1988 when the global action was initiated [4,5], but as the Bill and Melinda Gates foundation enthused, the last $1 \%$ is the most important and crucial in the final quest to rid the world of polio [6].

Currently, amongst the giant strides recorded by the coordinated push for polio eradication, type 3 and type 2 wild polio virus serotypes are no longer in circulation. The last case 
of paralytic poliomyelitis caused by type 2 wild polio virus was in $1999[5,7]$, while the last case by type 3 wild polio virus was in 2012 [5]. This leaves type 1 the lone wild type polio virus still in circulation.

However, incidences of circulating Vaccine Derived Paralytic Poliovirus type 2 (cVDPP2) have continued to be detected in Afghanistan, Pakistan, Nigeria and some other countries [8].

\subsection{Virology}

Poliomyelitis is an acute communicable disease caused by the wide poliovirus (WPV), a human enterovirus of the Picornaviridae family [9]. Poliovirus has a single-stranded, positive-sense RNA genome and a capsid of protein. The capsid is composed of 60 copies each of four proteins (VP1, VP2, VP3, and VP4) that form an icosahedral structure [10]. Poliovirus infection is initiated by the attachment of the virus to specific receptors called Pvr (poliovirus receptor) or CD155 [11]. The replication of poliovirus has been excellently reviewed in some recent studies [12, 13]. Two types of outcomes result from poliovirus infection, one that does not result in any symptoms and in which the central nervous system (CNS) is not affected and the other that result in symptoms and usually involves infection of CNS. Only about $1 \%$ of infections result in clinical symptoms [10]. The virus is made up of three serogroups that differ genetically (polio virus 1, polio virus 2 and polio virus 3 ). The uniqueness of the serogroups play important roles in the formulation of vaccines used in the prevention of the disease, as there is currently no cure. Transmission of the virus is mainly through the fecal-oral route or the oral contact with secretions from an infected individual [9].

\subsection{Vaccines}

The vaccines currently in use for polio prevention include the Sabin vaccine otherwise called the Oral Polio Vaccine (OPV) and the Salk vaccine or Inactivated Polio Vaccine (IPV). The two types of vaccines have their advantages and limitations. OPV is the predominant vaccine used in combating polio. The OPV can be univalent, divalent or trivalent based on the number of polio virus serotypes it confers immunity against. Though, the tOPV provides immunity against type 1,2 and 3 wild type polio virus, the protection is not as strong as that conferred by uOPV.

The advantages of OPV include low cost, long lasting protection, ease of administration, and ability to induce passive immunity in a community [14]. The major limitation of OPV is the chance of inducing circulating vaccine derived poliovirus (cVDPV). Although, the rate of occurrence is very low ( 1 in 2.7 million cases), this limitation still poses a serious risk especially in the end game to effectively rid the world of polio. Also, OPV provides the virus an avenue to re-enter the population by the reacquisition of virulence through genetic mutation.

IPV is mainly used in regions with a high standard of living and thus can afford the high cost of the vaccine. Such places include the USA that has been using IPV since 2001 [14]. There is though, a coordinated push to ensure that immunizations around the world are done with IPV to limit the Vaccine Derived Poliovirus (VDPV) persisting in the population.

\subsection{Epidemiology of Polio in Pakistan, Nigeria and Afghanistan}

The control of polio globally has been successful but the failure to interrupt the spread of the virus in Nigeria, Pakistan and Afghanistan remains a significant $\operatorname{cog}$ in the wheel of complete eradication of the virus. This threat can cause the emergence of 200,000 new cases of polio all over the world in less than 10 years [14]. This is possible as these three countries serve as nurseries from where the polio virus can spread to other parts of the world [1].

Although Nigeria is still categorized as one of the countries that have not interrupted the transmission of the polio virus, Pakistan and Afghanistan are the two remaining endemic countries and also are of extreme strategic importance in the fight against polio [1].

Over the past four years, the number of confirmed polio cases in Nigeria has recorded a significant decline. As at 2012, there were 122 cases, 2013 and 2014 saw the detection of 53 and 6 cases respectively, and zero case of polio virus infection in 2015 [15]. There was a resurgence of polio virus in Nigeria when 4 cases were detected in 2016, setting back the date for the certification of the country as completely polio free. The four cases were caused by the wild polio virus type 1 and occurred in 3 Local Government Areas (LGAs) in Borno state, Northeastern Nigeria. An important feature to note in these cases is that all of them occurred in Internally Displaced Persons (IDPs) from hitherto unreachable localities [15]. As at November 2017, there has been no recorded case of polio virus infection or positive test of an environmental sample in the country.

Afghanistan reported three wild poliovirus type 1 cases in the third quarter of 2016. The total number of polio cases in 2016 was 9. Importantly, there was detection of Vaccine-Derived Polio virus type 2 (VDPV2) which occurred in the Paktita province, thus heightening fears of the reemergence of the type 2 poliovirus which was considered eradicated in 1999. Unlike the situation in Nigeria in which the WPV1 transmission is localized within a small geographical area, the transmission in Afghanistan is spread across the Kunar province in the east, Northern parts of Helmand, Kandahar in the south and Bermel in the south-east. There have been four cases of WPV1 in 2017, with the most recent case being in April when an onset of paralysis occurred in Nawzad district, Helmand province. Also, environmental samples analyzed yielded six WPV1 positive samples with $50 \%$ of the positive samples originating from Kandahar province [16].

In Pakistan, there has only been one occurrence of polio virus in 2017, when a four year old tested positive in the Punjab province. The country has seen an erratic sequence in the number of detected polio cases in recent years. The number of polio cases though has seen a significant decline 
with only 20 cases recorded in 2016; compared to 54, 306 and 93 cases detected in 2015, 2014 and 2013 respectively. Pakistan has been described as the only prevalent polio reservoir and accounted for $80 \%$ of the global export of the virus [1].

\subsection{Acute Flaccid Paralysis (AFP) Surveillance}

One of the core strategies of the Global Polio Eradication Initiative is the Acute Flaccid Paralysis Surveillance. Acute Flaccid Paralysis (AFP) is defined as either "any case with recent or sudden onset of floppy paralysis or muscle weakness in a child aged less than 15 years of age, due to any cause; or "any paralytic illness in a person of any age in whom a clinician suspects polio" [17].

Due to the fact that individuals can be infected with polio virus and yet not present any symptoms ( $>90 \%$ ), effective surveillance to detect polio-like AFP cases is critical to achieving the goals of GPEI [18]. Surveillance of poliomyelitis is achieved by the proper reporting of AFP cases by healthcare providers. Environmental samples collected periodically for testing can also be used as supplement for the data provided by healthcare providers [5].

The AFP surveillance framework in Nigeria is structured to fit the political administration setup of the country (Nigeria is divided into 36 states, 1 Federal Capital Territory and 774 Local Government Areas). Every health facility in each LGA reports to the Disease Surveillance and Notification Officer (DSNO) in charge in the local government. The DSNO ensures the safe and prompt transport of the specimens collected to 1 of the 2 national laboratories present in the country [19]. The financial remuneration of the AFP staff is borne by WHO and other donor agencies such as the Bill and Melinda Gates foundation [18, 20].

In Afghanistan, AFP surveillance is done by staff of the WHO. These field workers called the Provincial Polio Officers (PPOs) work directly under the supervision of the Regional Polio Officers (RPOs) and Assistant Regional Polio Officers (ARPOs). The AFP surveillance is integrated into an already existing epidemiological surveillance system: Disease Early Warning System (DEWS) [21].

In Pakistan, AFP surveillance began in 1997 but it was not till 2000 before adequate attention was directed towards the program. The AFP surveillance system in Pakistan is functional at national, provincial and district levels. Healthcare providers or the community report any suspected case of AFP and surveillance officers also conduct weekly visits to priority facilities and other formal and informal healthcare facilities. Any sample collected is sent to the National Institute of Health (NIH) in Islamabad for analysis [22].

\section{Major Factors Limiting the Complete Eradication of Polio in the Three Countries}

Major hurdles have been identified that militate against the eradication of polio in these endemic countries. They include:

\subsection{Low Literacy Rate}

Pakistan and Nigeria are densely populated countries. Pakistan ranks the $6^{\text {th }}$ most populous country in the world with its 180 million citizens, while Nigeria has a population of 182 million people [23]. Although Afghanistan boasts a smaller population size of 34 million, a key similarity amongst the three countries is the prevalent low literacy rates compared to global standards. While Afghanistan has a national literacy rate of $38.2 \%$, the corresponding values are $58 \%$ and $55 \%$ in Pakistan and Nigeria respectively [24]. There is a positive correlation between low literacy rates and consequent vaccine rejection [14]. This constraint is exemplified in Pakistan, where the lowest number of polio cases is in Punjab which boasts a relatively higher literacy rate compared to other provinces such as the Federal Administered Tribal Areas (FATA) and Khyber Pakhtunkwa (KPK), where majority of the 306 cases reported for 2015 emanated [14].

\subsection{Conflict}

This is perhaps the most important hindrance to complete polio eradication in Pakistan, Nigeria and Afghanistan. Counter insurgencies in Pakistan in May 2011 gave rise to a spike in polio transmission leading to 198 detected cases in that year, while similar conflict action in 2014 saw the reported cases peaking at 306 compared to the 54 cases recorded in the previous year [25].

Ongoing conflict in FATA of Pakistan and southern Afghanistan currently hinders the reach of vaccinators to the vulnerable children in the area [8]. Sometimes, there is cancellation of proposed vaccination routines which significantly affects the overall effectiveness of the eradication program.

Nigeria has been experiencing insurgency in the north-eastern part of the country since 2009 with systematic target of health facilities [26]. The conflict in the region has so far led to 7 million of the 14.8 million in the area being displaced with 3.7 million in need of urgent healthcare interventions. More worrisome is the fact that $59 \%$ of the people in need of urgent health care intervention are inaccessible due to the conflict. The conflict in the region poses additional danger when the possibility of the virus spreading to neighboring regions such as Lake Chad, Northern Cameroon, Southern Niger and parts of the Central African Republic is factored in $[2,27]$.

Also, it is important not to underestimate the ability of militants present in these restive regions to fuel the spread of the virus. These militants are highly mobile and may be asymptomatic thus shedding the virus into the environment undetected [28]. These militants also serve as vehicles for the export of the virus to other parts of the world where their services are needed in the promotion of conflict. A typical example is the case of Xinjang province in China, close to the Pakistani border. In 2011, the province was restive and in a country where the last detected case of polio was in 1994, there was confirmation of seven paralytic polio cases. The strain of the virus implicated was WPV1, and after genetic 
profiling, it was discovered it bore strong semblance to the circulating strain present in Pakistan at the time [28].

\subsection{Vaccine Skepticism}

The refusal of parents or guardians to allow their wards to receive immunization is another major issue undermining the fight against polio eradication [29]. So many factors account for the lack of trust in immunization. One could highlight VDPV cases as a strong point for the rejection of immunization. Here some of the naysayers refuse based on the theory that the program is a coordinated attempt by Westerners to make them impotent.

Vaccine skepticism is a public health issue of global importance. It is vital because it affects the effectiveness of the global polio eradication initiative and other immunization campaigns [30]. Understanding how vaccine skepticism affects immunization strategies is important for the success of the global polio end game [31].

From 2012, there has been an upward trend in vaccine hesitancy owing to waning vaccine confidence. This is significant because the lowering of herd immunity in a population can lead to resurgence of polio even in certified polio-free populations [32].

Interestingly, vaccine skepticism is more prevalent in Europe compared to the remaining polio-endemic countries (Nigeria, Afghanistan and Pakistan). According to a study done by Larson et al. [31] comprising data from Georgia, India, Pakistan, UK and Nigeria; they found that Georgia had the highest number of vaccine refusal. Also, in another study carried out by other researchers in 2016 involving 67 countries, they reported vaccine skepticism of $41 \%$ and $36 \%$ for France and Bosnia \& Herzegovina respectively which were the highest amongst the countries studied. They concluded that there was an inverse relationship between socio economic status and level of positive vaccine sentiment [30].

It is of importance to note that vaccine skepticism is not peculiar to polio immunization alone. A report compiled by the European Union showed a steady increase in measles vaccine hesitation in some European countries such as Greece, Italy and Romania leading to reduced herd immunity and concomitant increase in measles cases [33].

Reasons given for the increase in vaccine skepticism include vaccine-side effects and vaccine safety [34]. For instance, in France, there has been multiple vaccine related controversies that have dampened vaccine confidence amongst the populace. In Nigeria, Pakistan and Afghanistan, vaccine skepticism is linked to the unfounded theory that immunization is a coordinated attempt by westerners to render their citizens impotent. Also, lack of adequate awareness on the advantages of getting vaccinated and vaccine-related misconceptions such as the ability of polio-related paralysis to be cured by herbal therapy; exposure of lone females to the alleged immoral advances from male vaccinators and the possibility of vaccination teams being spies of foreign governments [6], also play significant roles in fueling decrease in vaccine confidence.

A glimpse of the mammoth effect that vaccine refusal can have on the transmission of polio was seen in 2003, when an increased rate of vaccine skepticism occasioned by unverified rumors led to the suspension of vaccination in Kano state of northern Nigeria. The number of polio outbreaks skyrocketed leading to the export of the virus to 20 previously polio-free countries [27].

\subsection{Poor Healthcare System}

Pakistan, Afghanistan and Nigeria boast a healthcare delivery system that requires massive revitalization in order for it to meet the challenges it currently faces. Polio eradication has been limited due to this constraint. Although a lot of effort has been put into equipping the healthcare system to actively combat the polio scourge in the three countries courtesy of international organizations, there are still loopholes that aid the continued persistence of the virus in these regions. Ghafoor and Sheikh [29] outline such loopholes to include

"...flaws in health system (which) allow bundles of corruption both morally and financially resulting in stealing of resources. Absence of staff from duty, lack of field operations, and even use of vaccines for privately run clinics (which) affects service delivery in terms of quantity and quality..."

In addition to the loopholes outlined above, there are also issues of sub-optimal financing for these health institutions thus personnel are underpaid and lack some of the requisite materials needed to discharge their duties. In the conflict ridden state of Borno, North-eastern Nigeria, only one third of the health facilities still remain undamaged in the ongoing conflict. Out of the undamaged facilities, $33 \%$ are partially or fully dysfunctional [20].

\section{Recommendations and Possible Way Out}

The target of complete elimination of polio globally is not unachievable. There are though some important steps that can be initiated or their practice intensified before a polio-free world can become a certainty. These include:

i. Adequate sensitization should be carried out to dispel rumours and misconceptions of the polio campaign, especially in rural areas. Such sensitization should be done using local influential personalities.

ii. The vaccination campaign should be factored in when truce negotiations are being conducted in conflict prone regions. It should be actively discussed in order to reduce cases of inaccessibility of vulnerable populations who conveniently serve as incubating hubs for the virus.

iii. The issue of cVDPV (Circulating Vaccine Derived Polio Virus) should be effectively tackled as this poses significant risk to the successful elimination of the virus $[35,36]$. The synchronization of withdrawal of oral polio vaccine and the subsequent introduction of inactivated polio vaccine is pivotal to achieving this aim [37]. The high cost of IPV compared to OPV is a strong determinant for the success of this synchronization and as such, funding bodies, governments 
and individuals need to be more committed financially and materially.

iv. Adequate surveillance of the polio campaign cannot be overstated. This will aid the effective monitoring of immunization campaigns, detection of high risk areas and possible routes of spread of the virus.

$\mathrm{v}$. The overall success of polio eradication campaigns hinge to a large extent on the vaccination teams (health personnel and volunteers), and as such, their security should be paramount, especially as the regions where polio cases are still entrenched are conflict-prone places.

\section{Conclusions}

The eradication of polio globally is achievable. The end game is very important to this cause and as such maximum attention should be given to the three remaining hotspots of the virus (Nigeria, Afghanistan and Pakistan). Conflict is the major factor limiting the elimination of the virus in these three countries and thus programs in the polio eradication campaign should be streamlined to circumvent this threat.

\section{References}

[1] H. Yasin, "Polio: the fight of the public against a disease it does not understand with a vaccine it does not trust," J Pak Med Assoc, vol. 66, no. 5, 2016, pp. 496-497.

[2] L. Roberts, "New polio cases in Nigeria spur massive response," Science, vol. 353, no. 6301, 2016, p. 738. doi: $10.1126 /$ science. 353.6301 .738

[3] World Health Organization, "Government of Nigeria reports 2 wild polio cases, first since July 2014," Media Centre Geneva, Switzerland, 2016.

[4] E. K. Moturi, K. A. Porter, S. G. F. Wassilak, R. H. Tangermann, O. M. Diop, C. C. Burns, and H. Jafari, "Progress towards polio eradication - worldwide, 2013 - 2014," Morb Mort Wkly Rep Wkly, vol. 63, no. 21,2014 , pp. 468-472.

[5] I. M. Blake, P. Chenoweth, H. Okayasu, C. A. Donnelly, R. B. Alyward, and N. C. Grassly, "Faster Detection of Poliomyelitis Outbreaks to Support Polio Eradication," Emerg Infect Dis, vol. 22, no. 3, 2016, pp. 449-456.

[6] F. S. Hussain, P. Boyle, P. Patel, and R. Sullivan, "Eradicating polio in Pakistan: an analysis of the challenges and solutions to this security and health issue," Glob Health, vol. 12, 2016, pp. 63-71. doi: 10.1186/s12992-016-0195-3.

[7] B. Aylward, and T. Yamada, "The polio endgame," New Engl J Med, vol. 364, no. 24, 2011, pp. 2273-2275.

[8] N. C. Grassly, "The final stages of the global eradication of poliomyelitis. Phil Trans R Soc B, vol. 368, 2013, pp. 1-14. doi: 10.1098/rstb.2012.0140

[9] B. E. Bassey, R. G. Vaz, F. Braka, S. T. Komakech, S. T. Maleghemi, G. U. Akpan, and A. Ejeka-Okocha, "Profiling the immunity status of children with non-polio acute flaccid paralysis who had not received any doses of oral polio vaccine in the South-South region, Nigeria 2011-2014," Pub Health, vol. 139, 2016, pp. 148-153. doi: 10.1016/j.puhe.2016.05.009.
[10] V. R. Racaniello, "Picornaviridae: the viruses and their replication", In: D. M. Knipe, P. M. Howley, D. E. Griffin, R. A Lamb, M. A. Martin, B. Roizman, and S. E. Straus, (ed). Fields Virology, $4^{\text {th }}$ ed, vol. 1, Lippincott/the Williams \& Wilkins Co, New York, 2001, pp. 685-722.

[11] M. Strauss, D. J. Filman, D. M. Belnap, N. Cheng, R. T. Noel, and J. M. Hogle, "Nectin- like interactions between poliovirus and its receptor trigger conformational changes associated with cell entry," J Virol, vol. 89, no. 8, 2015, pp. 4143-4157.

[12] L. Romero-Brey, and R. Bartenschlager, "Membranous replication factories induced by plus-strand RNA viruses," Viruses, vol. 6, 2014, pp. 2826-2857.

[13] E. D. Rossignol, J. E. Yang, and E. Bullitt, “The role of electron microscopy in studying the continuum of changes in membranous structures during poliovirus infection," Viruses, vol. 7, 2015, pp. 5305-5318.

[14] M. Kabir, and M. S. Afzal, "Epidemiology of polio virus infection in Pakistan and possible risk factors for its transmission,” Asian Pac J Trop Med, vol. 352, 2016, pp. 1-4. doi: 10.1016/j.apjtm.2016.09.006.

[15] National Primary Health Care Development Agency, "Nigeria Polio Eradication Emergency Plan,” 2017.

[16] UNICEF, “Afghanistan Polio Update July - September 2016”, 2016.

[17] WHO, "WHO-recommended standards for surveillance of selected vaccine preventable diseases," Department of Vaccines and Biologicals Geneva, Switzerland, 2003.

[18] E. A. Coates, S. Waisbord, J. Awake, R. Solomon, and R. Dey, "Successful polio eradication in Uttar Pradesh, India: the pivotal contribution of the Social Mobilization Network, an NGO/UNICEF collaboration," Glob Health: Sc and Pract vol. 1, no. 1, 2013, pp. 63-68.

[19] S. G. F. Wassilak, C. L. Williams, S. C. Mursill, A. B. Dahl, C. Ohuabunwo, and R. H. Tangermann, "Using Acute Flaccid Paralysis Surveillance as a Platform for Vaccine- Preventable Disease Surveillance,” The J Infect Dis, vol. 216, 2017, pp. 293-298.

[20] WHO, "Polio eradication: experts say Nigeria not 'out of the woods yet'," Geneva, Switzerland, 2017. http://www.afro.who.int/news/

[21] Polio eradication.org, "External Review of Acute Flaccid Paralysis Surveillance for Polio Eradication in Four Regions of Afghanistan," 2017. Retrieved from http://polioeradication.org/uploads/2017/04

[22] End Polio Pakistan, "Surveillance," 2017. Retrieved from http://www.endpolio.com.pk/polioin-pakistan/surveillance

[23] National Population Commission, "Nigeria population now 182 million-NPC," Nigeria Population Commission (NPopC), 2017.

[24] UNESCO, "Literacy statistics metadata information table," UNESCO Institute for Statistics, September 2015. Retrieved December 2017.

[25] J. J. R. Bigna, "Polio eradication efforts in regions of geopolitical strife: the Boko Haram threat to efforts in sub-Saharan Africa," Afr Health Sc, vol. 16, no. 2, 2016, pp. 584-587. doi:10.4314/abs.v16i2.28. 
[26] D. Ali, R. Banda, A. Mohammed, J. Adagadzu, B. Murele, R. Seruyane, J. Makam, P. Mkanda, B. Okpessen, S. G. Tegegne, A. S. Folorunsho, T. B. Erbeto, Y. G. Yehualashet, and R. G. Vaz, "Strengthening routine immunization in areas of northern Nigeria at high risk of polio transmission during 2012-2014," J Infect Dis, vol. 213, 2016, pp. 147-150.

[27] N. U. Nasir, S. A. Bandyopadhyay, F. Mntagnani, E. J. Akite, B E. Mungu, V. I. Uche, and M. A. Ismaila, "Polio elimination in Nigeria: A review," Human Vac Immunotherap, vol. 12, no. 3, 2016, pp. 658-663.

[28] W. U. Z. Tariq, "Poliomyelitis, security issues and Pakistan," J Human Virol Retrovirol, vol. 5, no. 4, 2017, pp. 1-2.

[29] S. Ghafoor, and N. Sheikh, "Eradication and current status of poliomyelitis in Pakistan: Ground realities," J Immunol Res, 2016, doi: 10.1155/2016/6837824.

[30] H. J. Larson, W. S. Schulz, J. D. Tucker, and D. M. D. Smith, "Measuring vaccine confidence: introducing a global vaccine confidence index," PLoS Curr Outbreaks, 2015, doi: 10.1371/currents.outbreaks.ce0f6177.4.

[31] H. J. Larson, A. Figueredo, and S. N. Jones, "The state of vaccine confidence 2016: Global insights through a 67-country survey," EBioMed, vol. 12, 2016, pp. 295-301.

[32] WHO, "Report of the SAGE working group on vaccine hesitancy," 2014. Retrieved from http://www.who.int/immunization/sage/meetings/2014/october /1_Report_WORKING_GROUP vaccine_hesitancy_final.pdf

[33] EU, "Measles deaths in EU on course to treble," 2017, Retrieved from http://www.euronews.com/2017/12/06/measles-deaths-in-eu-o n-course-to-treble

[34] O. Yaqub, S. Castle-Clarke, N. Sevdalis, and J. Chataway, "Attitudes to vaccination: a critical review," Soc Sc Medline, vol. 112, 2014, pp. 1-11.

[35] M. Pons-Salort, C. C. Burns, H. Lyons, I. M. Blake, H. Jafari, M. S. Oberste, O. M. Kew, and N. C. Grassly, "Preventing vaccine-derived poliovirus emergence during the polio endgame," PLoS Pathogens vol. 12, no. 7, 2016, e1005728. doi: 10.1371/journal.ppat.1005728.

[36] G. Sherriff, Z. W. Mufti, G. R. Vaz, W. R. Sutter, and N. C. Grassly, "Estimated effect of inactivated poliovirus vaccine campaigns, Nigeria and Pakistan, January 2014- April 2016," Emerg Infect Dis vol. 23, no. 2, 2017, pp. 258-263. doi: org/10.3201/eid2302.161210.

[37] R. J. D. Tebbens, L. M. Hampton, and K. M. Thompson, "Implementation of coordinated global serotype 2 oral poliovirus vaccine cessation: risks of inadvertent trivalent oral poliovirus vaccine use," BMC Infect Dis vol. 16: 2016, pp. 237-247. doi: 10.1186/s12879-016-1537-8. 